\title{
An implicitly defined iterative sequence for monotone operators in Banach spaces
}

\section{Fumiaki Kohsaka*}

\section{"Correspondence:}

f-kohsaka@oita-u.ac.jp

Department of Computer Science

and Intelligent Systems, Oita

University, Dannoharu, Oita-shi,

Oita, 870-1192, Japan

\begin{abstract}
Given a monotone operator in a Banach space, we show that an iterative sequence, which is implicitly defined by a fixed point theorem for mappings of firmly nonexpansive type, converges strongly to a minimum norm zero point of the given operator. Applications to a convex minimization problem and a variational inequality problem are also included.
\end{abstract}

MSC: $47 \mathrm{H} 05 ; 47 \mathrm{~J} 25 ; 47 \mathrm{~N} 10$

Keywords: Banach space; convex minimization; mapping of firmly nonexpansive type; fixed point; monotone operator; proximal point method; variational inequality

\section{Introduction}

Many nonlinear problems can be formulated as the problem of finding a zero point of a maximal monotone operator in a Banach space. The proximal point method, which was first introduced by Martinet [1] and generally studied by Rockafellar [2], is an iterative method for approximating a solution to this problem.

The proximal point method generates a sequence $\left\{x_{n}\right\}$ by $x_{1} \in X$ and

$$
x_{n+1}=\left(J+\lambda_{n} A\right)^{-1} J x_{n}
$$

for all $n \in \mathbb{N}$, where $A: X \rightarrow 2^{X^{*}}$ is a maximal monotone operator, $X$ is a smooth, strictly convex, and reflexive real Banach space, $J: X \rightarrow X^{*}$ is the normalized duality mapping, and $\left\{\lambda_{n}\right\}$ is a sequence of positive real numbers.

The following result was obtained in [3]: If $\sum_{n=1}^{\infty} \lambda_{n}=\infty$, then $\left\{x_{n}\right\}$ is bounded if and only if $A^{-1} 0$ is nonempty. Further, if $X$ is uniformly convex, the norm of $X$ is uniformly Gâteaux differentiable, $A^{-1} 0$ is nonempty, $\inf _{n} \lambda_{n}>0$, and $J$ is weakly sequentially continuous, then $\left\{x_{n}\right\}$ converges weakly to an element of $A^{-1} 0$. This is a generalization of the result due to Rockafellar [2] in Hilbert spaces. See also [4, 5] for some related results.

The aim of the present paper is to study the asymptotic behavior of the sequence $\left\{x_{n}\right\}$ generated by

$$
x_{n}=\beta_{n}\left(J+\lambda_{n} A\right)^{-1} J x_{n}
$$

for all $n \in \mathbb{N}$, where $A, X, J$, and $\left\{\lambda_{n}\right\}$ are the same as in (1.1) and $\left\{\beta_{n}\right\}$ is a sequence of $[0,1)$. Under some additional assumptions, we show that $\left\{x_{n}\right\}$ is well defined and is strongly convergent to an element of $A^{-1} 0$ of minimal norm; see Theorem 3.5.

○2014 Kohsaka; licensee Springer. This is an Open Access article distributed under the terms of the Creative Commons Attribution License (http://creativecommons.org/licenses/by/2.0), which permits unrestricted use, distribution, and reproduction in any medium, provided the original work is properly cited. 
The schemes (1.1) and (1.2) above are similar to each other, though their properties are quite different. In fact, the former fails to converge strongly even in Hilbert spaces [6], whereas the latter converges strongly in Banach spaces. Further, the former is well defined since $\mathrm{R}(J+\lambda A)=X^{*}$ for each $\lambda>0$, whereas the latter is not necessarily well defined. To study the well definedness and the asymptotic behavior of $\left\{x_{n}\right\}$ in (1.2), we exploit some techniques in $[3,7]$.

This paper is organized as follows: In Section 2, we give some definitions, recall some known results, and briefly study the existence of a zero point of a monotone operator. In Section 3, using the results in the previous section, we first obtain a convergence theorem for a monotone operator satisfying a range condition; see Theorem 3.1. Using this result, we show a convergence theorem for a maximal monotone operator; see Theorem 3.5. In Section 4, we apply Theorem 3.5 to a convex minimization problem and a variational inequality problem.

\section{Preliminaries}

Throughout the present paper, we denote by $\mathbb{N}$ the set of all positive integers, $\mathbb{R}$ the set of all real numbers, $X$ a smooth, strictly convex, and reflexive real Banach space with dual $X^{*}$, $\|\cdot\|$ the norms of $X$ and $X^{*},\left\langle x, x^{*}\right\rangle$ the value of $x^{*} \in X^{*}$ at $x \in X, x_{n} \rightarrow x$ the strong convergence of a sequence $\left\{x_{n}\right\}$ of $X$ to $x \in X, x_{n} \rightarrow x$ the weak convergence of a sequence $\left\{x_{n}\right\}$ of $X$ to $x \in X, \bar{U}$ the norm closure of $U \subset X, \operatorname{co} U$ the convex hull of $U \subset X, \overline{c o} U$ the closed convex hull of $U \subset X$, and $S_{X}$ the unit sphere of $X$, respectively.

Under the assumptions on $X$, we know that for each $x \in X$, there is a corresponding unique $J x$ in $X^{*}$ such that $\langle x, J x\rangle=\|x\|^{2}$ and $\|J x\|=\|x\|$. The mapping $J$ is called the normalized duality mapping of $X$ into $X^{*}$. We know the following: $J: X \rightarrow X^{*}$ is a bijection; $J(\alpha x)=\alpha J x$ for all $\alpha \in \mathbb{R}$ and $x \in X$; $J$ is norm-to-weak continuous, that is, $J x_{n} \rightarrow J x$ whenever $\left\{x_{n}\right\}$ is a sequence of $X$ such that $x_{n} \rightarrow x \in X$; $J$ is strictly monotone, that is, $\left\langle x-y, x^{*}-y^{*}\right\rangle>0$ for all distinct $x, y \in X$. The norm of $X$ is said to be uniformly Gâteaux differentiable if the limit

$$
\lim _{t \rightarrow 0} \frac{\|x+t y\|-\|x\|}{t}
$$

converges uniformly in $x \in S_{X}$ for all $y \in S_{X}$. The space $X$ is said to be uniformly convex if for each $\varepsilon \in(0,2]$, there exists $\delta>0$ such that $\|(x+y) / 2\| \leq 1-\delta$ whenever $x, y \in S_{X}$ and $\|x-y\| \geq \varepsilon$. The space $X$ is said to have the Kadec-Klee property if $x_{n} \rightarrow x$ whenever $\left\{x_{n}\right\}$ is a sequence of $X$ such that $x_{n} \rightarrow x \in X$ and $\left\|x_{n}\right\| \rightarrow\|x\|$. Every uniformly convex Banach space is both strictly convex and reflexive and has the Kadec-Klee property; see [8, 9].

For a nonempty closed convex subset $C$ of $X$ and $x \in X$, there exists a unique point $\hat{x}$ in $C$ such that $\|\hat{x}-x\| \leq\|y-x\|$ for all $y \in C$. The metric projection $P_{C}$ of $X$ onto $C$ is defined by $P_{C} x=\hat{x}$ for all $x \in X$. It is well known [9] that

$$
z=P_{C}(x) \quad \Longleftrightarrow \quad \sup _{y \in C}\langle y-z, J(x-z)\rangle \leq 0
$$

for $(x, z) \in X \times C$. The function $\phi: X \times X \rightarrow \mathbb{R}$ is defined by

$$
\phi(x, y)=\|x\|^{2}-2\langle x, J y\rangle+\|y\|^{2}
$$


for all $x, y \in X$; see $[10,11]$. If $X$ is a Hilbert space, then $\phi(x, y)=\|x-y\|^{2}$ for all $x, y \in X$. It is easy to see that

$$
(\|x\|-\|y\|)^{2} \leq \phi(x, y)
$$

and

$$
\phi(x, y)+\phi(y, x)=2\langle x-y, J x-J y\rangle
$$

for all $x, y \in X$.

Let $C$ be a nonempty subset of $X$ and $T: C \rightarrow X$ a mapping. The set of all fixed points of $T$ is denoted by $F(T)$. The mapping $T$ is said to be of firmly nonexpansive type [12] if

$$
\langle T x-T y, J T x-J T y\rangle \leq\langle T x-T y, J x-J y\rangle
$$

for all $x, y \in C$; see also [13]. If $X$ is a Hilbert space, then $T: C \rightarrow X$ is firmly nonexpansive if and only if it is of firmly nonexpansive type.

For an operator $A: X \rightarrow 2^{X^{*}}$, the domain $\mathrm{D}(A)$, the range $\mathrm{R}(A)$, and the graph $\mathrm{G}(A)$ of $A$ are defined by $\mathrm{D}(A)=\{x \in X: A x \neq \emptyset\}, \mathrm{R}(A)=\bigcup_{x \in X} A x$, and $\mathrm{G}(A)=\left\{\left(x, x^{*}\right) \in X \times\right.$ $\left.X^{*}: x^{*} \in A x\right\}$, respectively. The operator $A$ is said to be monotone if $\left\langle x-y, x^{*}-y^{*}\right\rangle \geq 0$ whenever $\left(x, x^{*}\right),\left(y, y^{*}\right) \in \mathrm{G}(A)$. It is also said to be maximal monotone if $A$ is monotone and there is no monotone operator $B: X \rightarrow 2^{X^{*}}$ such that $A \neq B$ and $\mathrm{G}(A) \subset \mathrm{G}(B)$. Let $C$ be a nonempty closed convex subset of $X$ and $A: X \rightarrow 2^{X^{*}}$ a monotone operator such that $\mathrm{D}(A) \subset C \subset J^{-1} \mathrm{R}(J+A)$. Then the mapping $T: C \rightarrow C$ defined by $T x=(J+A)^{-1} J x$ for all $x \in C$ is of firmly nonexpansive type and $\mathrm{F}(T)=A^{-1} 0$; see $[12,14]$. We know the following lemma.

Lemma 2.1 ([3]) Suppose that the norm of $X$ is uniformly Gâteaux differentiable. Let $C$ be a nonempty closed convex subset of $X, A: X \rightarrow 2^{X^{*}}$ a monotone operator such that

$$
\mathrm{D}(A) \subset C \subset \bigcap_{\lambda>0} J^{-1} \mathrm{R}(J+\lambda A)
$$

$\left\{\lambda_{n}\right\}$ a sequence of $(0, \infty)$ such that $\inf _{n} \lambda_{n}>0$, and $Q_{\lambda_{n}}: C \rightarrow C$ the mapping defined by $Q_{\lambda_{n}} x=\left(J+\lambda_{n} A\right)^{-1} J x$ for all $x \in C$ and $n \in \mathbb{N}$. If $\left\{x_{n}\right\}$ is a sequence of $C$ such that $x_{n} \rightarrow u$ and $x_{n}-Q_{\lambda_{n}} x_{n} \rightarrow 0$, then $u$ is an element of $A^{-1} 0$.

We know the following result for mappings of firmly nonexpansive type.

Lemma 2.2 ([12]) Let $C$ be a nonempty closed convex subset of $X$ and $T: C \rightarrow C$ a mapping of firmly nonexpansive type. Then the following hold:

(i) $\mathrm{F}(T)$ is nonempty if and only if $\left\{T^{n} x\right\}$ is bounded for some $x \in C$;

(ii) $\mathrm{F}(T)$ is closed and convex.

Using Lemma 2.2, we can show the following.

Lemma 2.3 Let $C$ be a nonempty closed convex subset of $X$ and $T: C \rightarrow C$ a mapping of firmly nonexpansive type. Suppose that $\beta \in[0,1), 0 \in C$, and $T(C)$ is bounded. Then the mapping $\beta$ T has a unique fixed point. 
Proof Set $S=\beta T$. Since $J$ is monotone, $T$ is of firmly nonexpansive type, and $\beta \in[0,1)$, we have

$$
\begin{aligned}
0 & \leq\langle S x-S y, J S x-J S y\rangle=\beta^{2}\langle T x-T y, J T x-J T y\rangle \\
& \leq \beta^{2}\langle T x-T y, J x-J y\rangle \\
& =\beta\langle S x-S y, J x-J y\rangle \leq\langle S x-S y, J x-J y\rangle
\end{aligned}
$$

for all $x, y \in C$. This implies that $S$ is of firmly nonexpansive type. Since $C$ is convex, $0 \in C$, and $\beta \in[0,1)$, we know that $S$ is a mapping of $C$ into itself. Further, since $S(C)=\beta T(C)$ and $T(C)$ is bounded, the sequence $\left\{S^{n} x\right\}$ is bounded for all $x \in C$. Thus Lemma 2.2 implies that $\mathrm{F}(S)$ is nonempty.

We next show that $\mathrm{F}(S)$ consists of one point. Suppose that $p, p^{\prime} \in \mathrm{F}(S)$. Then it follows from (2.8) that $(1-\beta)\left\langle p-p^{\prime}, J p-J p^{\prime}\right\rangle=0$. Since $1-\beta>0$, we obtain $\left\langle p-p^{\prime}, J p-J p^{\prime}\right\rangle=0$. Thus the strict monotonicity of $J$ implies that $p=p^{\prime}$.

As a direct consequence of Lemmas 2.2 and 2.3, we obtain the following.

Corollary 2.4 Let $A: X \rightarrow 2^{X^{*}}$ be a monotone operator such that $\mathrm{D}(A)$ is bounded and $\mathrm{D}(A) \subset C \subset J^{-1} \mathrm{R}(J+A)$ for some nonempty closed convex subset $C$ of $X$. Then the following hold:

(i) $A^{-1} 0$ is nonempty, closed, and convex;

(ii) if $0 \in C$ and $\beta \in[0,1)$, then there exists a unique $p \in C$ such that

$$
p=\beta(J+A)^{-1} J p
$$

Proof Let $T: C \rightarrow C$ be the mapping defined by $T x=(J+A)^{-1} J x$ for all $x \in C$. Then we know that $T$ is of firmly nonexpansive type and $T(C) \subset \mathrm{D}(A)$. Hence $\left\{T^{n} x\right\}$ is bounded for all $x \in C$. On the other hand, we know that $\mathrm{F}(T)=A^{-1} 0$. Therefore, part (i) follows from Lemma 2.2. Part (ii) follows from Lemma 2.3.

\section{Strong convergence of an iterative sequence}

In this section, we first show the following strong convergence theorem for a monotone operator satisfying a range condition.

Theorem 3.1 Let $X$ be a smooth, strictly convex, and reflexive real Banach space, $C$ a nonempty closed convex subset of $X$ such that $0 \in C$, and $A: X \rightarrow 2^{X^{*}}$ a monotone operator such that $\mathrm{D}(A)$ is bounded and

$$
\mathrm{D}(A) \subset C \subset \bigcap_{\lambda>0} J^{-1} \mathrm{R}(J+\lambda A) .
$$

Let $\left\{\lambda_{n}\right\}$ be a sequence of positive real numbers, $\left\{\beta_{n}\right\}$ a sequence of $[0,1)$, and $Q_{\lambda_{n}}: C \rightarrow C$ the mapping defined by $Q_{\lambda_{n}} x=\left(J+\lambda_{n} A\right)^{-1} J x$ for all $x \in C$ and $n \in \mathbb{N}$. Then the following hold:

(i) For each $n \in \mathbb{N}$, there exists a unique $x_{n} \in C$ such that $x_{n}=\beta_{n} Q_{\lambda_{n}} x_{n}$;

(ii) if $X$ has the Kadec-Klee property, the norm of $X$ is uniformly Gâteaux differentiable, $\inf _{n} \lambda_{n}>0$, and $\lim _{n} \beta_{n}=1$, then the sequence $\left\{x_{n}\right\}$ converges strongly to $P_{A^{-1} 0}(0)$. 
Part (i) of Theorem 3.1 follows from Corollary 2.4.

The proof of (i) of Theorem 3.1 Let $n \in \mathbb{N}$ be given and set $B=\lambda_{n} A$. Then $B: X \rightarrow 2^{X^{*}}$ is monotone and $\mathrm{D}(B)=\mathrm{D}(A)$. Thus we know that $\mathrm{D}(B)$ is bounded and $\mathrm{D}(B) \subset C \subset J^{-1} \mathrm{R}(J+$ $B)$. Therefore, part (ii) of Corollary 2.4 ensures the conclusion.

Before proving (ii) of Theorem 3.1, we show the following lemma.

Lemma 3.2 The following hold:

(i) $\left\langle Q_{\lambda_{n}} x_{n}-y, J Q_{\lambda_{n}} x_{n}\right\rangle \leq 0$ for all $y \in A^{-1} 0$ and $n \in \mathbb{N}$;

(ii) $\phi\left(Q_{\lambda_{n}} x_{n}, y\right)+\phi\left(y, Q_{\lambda_{n}} x_{n}\right) \leq 2\left\langle y-Q_{\lambda_{n}} x_{n}, J y\right\rangle$ for all $y \in A^{-1} 0$ and $n \in \mathbb{N}$.

Proof We show (i). Let $y \in A^{-1} 0$ and $n \in \mathbb{N}$ be given. Since $Q_{\lambda_{n}}$ is of firmly nonexpansive type and $Q_{\lambda_{n}} y=y$, we know that

$$
\left\langle Q_{\lambda_{n}} x_{n}-y, J Q_{\lambda_{n}} x_{n}-J x_{n}\right\rangle \leq 0
$$

On the other hand, by the definition of $\left\{x_{n}\right\}$, we also know that

$$
J Q_{\lambda_{n}} x_{n}-J x_{n}=J Q_{\lambda_{n}} x_{n}-J\left(\beta_{n} Q_{\lambda_{n}} x_{n}\right)=\left(1-\beta_{n}\right) J Q_{\lambda_{n}} x_{n} .
$$

By (3.2), (3.3), and $1-\beta_{n}>0$, the result follows.

By (2.5) and (i), we have

$$
\begin{aligned}
\phi\left(Q_{\lambda_{n}} x_{n}, y\right)+\phi\left(y, Q_{\lambda_{n}} x_{n}\right) & =2\left\langle Q_{\lambda_{n}} x_{n}-y, J Q_{\lambda_{n}} x_{n}-J y\right\rangle \\
& \leq 2\left\langle Q_{\lambda_{n}} x_{n}-y,-J y\right\rangle
\end{aligned}
$$

for all $y \in A^{-1} 0$ and $n \in \mathbb{N}$. Thus the result follows.

We next show (ii) of Theorem 3.1.

The proof of (ii) of Theorem 3.1 Suppose that $X$ has the Kadec-Klee property, the norm of $X$ is uniformly Gâteaux differentiable, $\inf _{n} \lambda_{n}>0$, and $\lim _{n} \beta_{n}=1$. Set $y_{n}=Q_{\lambda_{n}} x_{n}$ for all $n \in \mathbb{N}$. By (i) of Corollary 2.4 , the set $A^{-1} 0$ is nonempty, closed, and convex. Hence $P_{A^{-1} 0}$ is well defined. We denote $P_{A^{-1} 0}$ by $P$.

Since $y_{n} \in \mathrm{D}(A)$ for all $n \in \mathbb{N}$ and $\mathrm{D}(A)$ is bounded, $\left\{y_{n}\right\}$ is bounded. By the definition of $\left\{x_{n}\right\}$, we have $\left\|x_{n}\right\|=\beta_{n}\left\|y_{n}\right\| \leq\left\|y_{n}\right\|$ for all $n \in \mathbb{N}$. Thus $\left\{x_{n}\right\}$ is also bounded.

Let $\left\{x_{n_{i}}\right\}$ be any subsequence of $\left\{x_{n}\right\}$. To see that $x_{n} \rightarrow P(0)$, it is sufficient to see that there exists a subsequence $\left\{x_{n_{i_{j}}}\right\}$ of $\left\{x_{n_{i}}\right\}$ which converges strongly to $P(0)$. Since $X$ is reflexive and $\left\{x_{n}\right\}$ is a bounded sequence of $C$, there exist $u \in C$ and a subsequence $\left\{x_{n_{i_{j}}}\right\}$ of $\left\{x_{n_{i}}\right\}$ such that $x_{n_{i_{j}}} \rightarrow u$. Since $\left\{y_{n}\right\}$ is bounded and $\lim _{n} \beta_{n}=1$, we have

$$
\left\|x_{n}-y_{n}\right\|=\left\|\beta_{n} y_{n}-y_{n}\right\|=\left(1-\beta_{n}\right)\left\|y_{n}\right\| \rightarrow 0 \text {. }
$$

Since $\inf _{j} \lambda_{n_{i j}} \geq \inf _{n} \lambda_{n}>0$, Lemma 2.1 shows that $u$ is an element of $A^{-1} 0$. It also follows from (3.5) and $x_{n_{i_{j}}} \rightarrow u$ that $y_{n_{i_{j}}} \rightarrow u$. 
We next show that $x_{n_{i j}} \rightarrow u$. By (2.4) and (ii) of Lemma 3.2, we have

$$
\begin{aligned}
\left(\left\|y_{n_{i_{j}}}\right\|-\|u\|\right)^{2} & \leq \phi\left(y_{n_{i_{j}}}, u\right) \\
& \leq \phi\left(y_{n_{i_{j}}}, u\right)+\phi\left(u, y_{n_{i_{j}}}\right) \leq 2\left\langle u-y_{n_{i_{j}}}, J u\right\rangle \rightarrow 0 .
\end{aligned}
$$

Thus we obtain $\left\|y_{n_{i_{j}}}\right\| \rightarrow\|u\|$. Since $X$ has the Kadec-Klee property, we have $y_{n_{i_{j}}} \rightarrow u$. Consequently, it follows from (3.5) that $x_{n_{i_{j}}} \rightarrow u$.

We next show that $u=P(0)$. To see this, let $y \in A^{-1} 0$ be given. Since $J$ is norm-to-weak continuous and $y_{n_{i_{j}}} \rightarrow u$, we know that $J y_{n_{i_{j}}} \rightarrow J u$. On the other hand, by (i) of Lemma 3.2, we have

$$
\left\langle y_{n_{i_{j}}}-y, J y_{n_{i_{j}}}\right\rangle \leq 0
$$

for all $j \in \mathbb{N}$. Letting $j \rightarrow \infty$ in (3.7), we obtain $\langle u-y, J u\rangle \leq 0$ and hence

$$
\sup _{y \in A^{-1} 0}\langle y-u, J(0-u)\rangle \leq 0
$$

Noting that $u \in A^{-1} 0$, we have from (2.2) and (3.8) that $u=P(0)$. Therefore, we conclude that $\left\{x_{n}\right\}$ converges strongly to $P(0)$.

As a direct consequence of Theorem 3.1, we obtain the following corollary.

Corollary 3.3 Let $X$ be a smooth, strictly convex, and reflexive real Banach space, $C$ a nonempty closed convex subset of $X$ such that $0 \in C, T: C \rightarrow C$ a mapping of firmly nonexpansive type such that $T(C)$ is bounded, $\left\{\lambda_{n}\right\}$ a sequence of positive real numbers, and $\left\{\beta_{n}\right\}$ a sequence of $[0,1)$. Then the following hold:

(i) For each $n \in \mathbb{N}$, there exists a unique $x_{n} \in C$ such that $x_{n}=\beta_{n} T x_{n}$;

(ii) if $X$ has the Kadec-Klee property, the norm of $X$ is uniformly Gâteaux differentiable, and $\lim _{n} \beta_{n}=1$, then the sequence $\left\{x_{n}\right\}$ converges strongly to $P_{\mathrm{F}(T)}(0)$.

Proof Let $A: X \rightarrow 2^{X^{*}}$ be the mapping defined by $A=J T^{-1}-J$, where $T^{-1}: X \rightarrow 2^{X}$ is defined by

$$
T^{-1} x= \begin{cases}\{u \in C: T u=x\} & (x \in T(C)) ; \\ \emptyset & (x \notin T(C))\end{cases}
$$

for all $x \in X$. Then, by [14], we know that the following hold:

- $A$ is a monotone operator and $A^{-1} 0=\mathrm{F}(T)$;

- $\mathrm{D}(A)=T(C) \subset C=J^{-1} \mathrm{R}(J+A)$;

- $T x=(J+A)^{-1} J x$ for all $x \in C$.

Thus the result follows from Theorem 3.1.

Remark 3.4 In the case when $C$ is bounded, Corollary 3.3 is reduced to the result obtained in [7]. 
Using Theorem 3.1, we can also show the following strong convergence theorem for a maximal monotone operator.

Theorem 3.5 Let $X$ be a smooth, strictly convex, and reflexive real Banach space, $A: X \rightarrow$ $2^{X^{*}}$ a maximal monotone operator such that $0 \in \overline{\mathrm{D}(A)}$ and $\mathrm{D}(A)$ is bounded, $\left\{\lambda_{n}\right\}$ a sequence of positive real numbers, and $\left\{\beta_{n}\right\}$ a sequence of $[0,1)$. Then for each $n \in \mathbb{N}$, there exists a unique $x_{n} \in \overline{\mathrm{D}(A)}$ satisfying (1.2). Moreover, if $X$ has the Kadec-Klee property, the norm of $X$ is uniformly Gâteaux differentiable, $\inf _{n} \lambda_{n}>0$, and $\lim _{n} \beta_{n}=1$, then the sequence $\left\{x_{n}\right\}$ converges strongly to $P_{A^{-1} 0}(0)$.

Proof The maximal monotonicity of $A$ implies that $\mathrm{D}(A)$ is nonempty and hence so is $\overline{\mathrm{D}(A)}$. It is obvious that $\overline{\mathrm{D}(A)}$ is closed. It is well known [15] that $\overline{\mathrm{D}(A)}$ is convex. In fact, we know that

$$
\lim _{\mu \downarrow 0}\left(I+\mu J^{-1} A\right)^{-1} x=x
$$

for all $x \in \overline{\mathrm{co}} \mathrm{D}(A)$, where $I$ denotes the identity mapping on $X$; see [16, 17]. Since $(I+$ $\left.\mu J^{-1} A\right)^{-1} x \in \mathrm{D}(A)$ for all $\mu>0$ and $x \in X$, it follows from (3.10) that $\overline{\operatorname{co}} \mathrm{D}(A) \subset \overline{\mathrm{D}(A)}$. Thus $\overline{\mathrm{co}} \mathrm{D}(A)=\overline{\mathrm{D}(A)}$ and hence $\overline{\mathrm{D}(A)}$ is convex.

On the other hand, since $A$ is maximal monotone, we know that $\mathrm{R}(J+\lambda A)=X^{*}$ for all $\lambda>0$; see [18]. Putting $C=\overline{\mathrm{D}(A)}$, we have

$$
\mathrm{D}(A) \subset C \subset X=\bigcap_{\lambda>0} J^{-1} \mathrm{R}(J+\lambda A)
$$

Noting that $0 \in \overline{\mathrm{D}(A)}=C$, we obtain the desired result by Theorem 3.1.

\section{Results deduced from Theorem 3.5}

In this final section, we study two applications of Theorem 3.5. Throughout this section, we suppose the following:

- $X$ is a uniformly convex real Banach space whose norm is uniformly Gâteaux differentiable;

- $\left\{\lambda_{n}\right\}$ is a sequence of positive real numbers such that $\inf _{n} \lambda_{n}>0$;

- $\left\{\beta_{n}\right\}$ is a sequence of $[0,1)$ such that $\lim _{n} \beta_{n}=1$.

We first study a convex minimization problem. For a function $f: X \rightarrow(-\infty, \infty]$, we denote by $\operatorname{argmin} f$ or $\operatorname{argmin}_{y \in X} f(y)$ the set of all $u \in X$ such that $f(u)=\inf f(X)$. In the case when $\operatorname{argmin} f=\{p\}$ for some $p \in X$, we identify $\operatorname{argmin} f$ with $p$. The set of all $x \in X$ such that $f(x) \in \mathbb{R}$ is denoted by $\mathrm{D}(f)$. We denote by $\partial f$ the subdifferential mapping of $f$; see $[17$, 19] for more details.

Corollary 4.1 Let $f: X \rightarrow(-\infty, \infty]$ be a proper lower semicontinuous convex function such that $0 \in \overline{\mathrm{D}(f)}$ and $\mathrm{D}(f)$ is bounded. Then for each $n \in \mathbb{N}$, there exists a unique $x_{n} \in \overline{\mathrm{D}(f)}$ such that

$$
x_{n}=\beta_{n} \underset{y \in X}{\operatorname{argmin}}\left\{f(y)+\frac{1}{2 \lambda_{n}} \phi\left(y, x_{n}\right)\right\} .
$$

Moreover, the sequence $\left\{x_{n}\right\}$ converges strongly to $P_{\operatorname{argmin} f}(0)$. 
Proof Let $A: X \rightarrow 2^{X^{*}}$ be the operator defined by $A=\partial f$. It is well known that $A$ is maximal monotone [20] and $A^{-1} 0=\operatorname{argmin} f$. Since $\mathrm{D}(f)$ is bounded and $\mathrm{D}(A) \subset \mathrm{D}(f)$, we know that $\mathrm{D}(A)$ is bounded. By Brøndsted and Rockafellar's theorem [21], we know that $\overline{\mathrm{D}(f)}=\overline{\mathrm{D}(A)}$. Thus we have $0 \in \overline{\mathrm{D}(A)}$. Further, the equality

$$
(J+\lambda A)^{-1} J x=\underset{y \in X}{\operatorname{argmin}}\left\{f(y)+\frac{1}{2 \lambda} \phi(y, x)\right\}
$$

holds for all $\lambda>0$ and $x \in X$. Thus we know that $x_{n}=\beta_{n}\left(J+\lambda_{n} A\right)^{-1} J x_{n}$ for all $n \in \mathbb{N}$. Consequently, Theorem 3.5 implies the conclusion.

We finally study a variational inequality problem. For a nonempty closed convex subset $C$ of $X$ and an operator $B: C \rightarrow X^{*}$, we denote by $\mathrm{VI}(C, B)$ the set of all $u \in C$ such that $\langle y-u, B u\rangle \geq 0$ for all $y \in C$. In the case when $\operatorname{VI}(C, B)=\{p\}$ for some $p \in C$, we identify $\operatorname{VI}(C, B)$ with $p$. The operator $B$ is said to be hemicontinuous if the mapping $g:[0,1] \rightarrow X^{*}$ defined by $g(t)=B(t x+(1-t) y)$ for all $t \in[0,1]$ is continuous with respect to the weak* topology in $X^{*}$ for all $x, y \in C$.

Corollary 4.2 Let $C$ be a nonempty bounded closed convex subset of $X$ such that $0 \in C$ and $B: C \rightarrow X^{*}$ a monotone and hemicontinuous operator. Then for each $n \in \mathbb{N}$, there exists a unique $x_{n} \in C$ such that

$$
x_{n}=\beta_{n} \operatorname{VI}\left(C, B+\frac{1}{\lambda_{n}}\left(J-J x_{n}\right)\right) .
$$

Moreover, the sequence $\left\{x_{n}\right\}$ converges strongly to $P_{\mathrm{VI}(C, B)}(0)$.

Proof Let $A: X \rightarrow 2^{X^{*}}$ be the operator defined by

$$
A(x)= \begin{cases}B(x)+\partial i_{C}(x) & (x \in C) ; \\ \emptyset & (x \in X \backslash C)\end{cases}
$$

for all $x \in X$, where $i_{C}$ denotes the indicator function of $C$. It is well known that $A$ is maximal monotone [18], $A^{-1} 0=\mathrm{VI}(C, B)$, and $\mathrm{D}(A)=C$. Thus we know that $\mathrm{D}(A)$ is bounded and $0 \in \mathrm{D}(A)$. Further, the equality

$$
(J+\lambda A)^{-1} J x=\mathrm{VI}\left(C, B+\frac{1}{\lambda}(J-J x)\right)
$$

holds for all $\lambda>0$ and $x \in X$. Thus we have $x_{n}=\beta_{n}\left(J+\lambda_{n} A\right)^{-1} J x_{n}$ for all $n \in \mathbb{N}$. Consequently, Theorem 3.5 implies the conclusion.

Competing interests

The author declares that he has no competing interests.

\section{Acknowledgements}

The author would like to thank the anonymous referees for carefully reading the original version of the manuscript. The author is supported by Grant-in-Aid for Young Scientists No. 25800094 from the Japan Society for the Promotion of Science. 


\section{References}

1. Martinet, B: Régularisation d'inéquations variationnelles par approximations successives. Rev. Fr. Inform. Rech. Opér. 4, 154-158 (1970) (in French)

2. Rockafellar, RT: Monotone operators and the proximal point algorithm. SIAM J. Control Optim. 14, $877-898$ (1976)

3. Aoyama, K, Kohsaka, F, Takahashi, W: Proximal point methods for monotone operators in Banach spaces. Taiwanese J. Math. 15, 259-281 (2011)

4. Kamimura, S: The proximal point algorithm in a Banach space. In: Nonlinear Analysis and Convex Analysis, pp. 143-148. Yokohama Publishers, Yokohama (2004)

5. Kamimura, S, Kohsaka, F, Takahashi, W: Weak and strong convergence theorems for maximal monotone operators in a Banach space. Set-Valued Anal. 12, 417-429 (2004)

6. Güler, O: On the convergence of the proximal point algorithm for convex minimization. SIAM J. Control Optim. 29, 403-419 (1991)

7. Kohsaka, F, Takahashi, W: Strongly convergent net given by a fixed point theorem for firmly nonexpansive type mappings. Appl. Math. Comput. 202, 760-765 (2008)

8. Cioranescu, I: Geometry of Banach Spaces, Duality Mappings and Nonlinear Problems. Kluwer Academic, Dordrecht (1990)

9. Takahashi, W: Nonlinear Functional Analysis. Yokohama Publishers, Yokohama (2000)

10. Alber, Yl: Metric and generalized projection operators in Banach spaces: properties and applications. In: Theory and Applications of Nonlinear Operators of Accretive and Monotone Type. Lecture Notes in Pure and Appl. Math., vol. 178, pp. 15-50. Dekker, New York (1996)

11. Kamimura, S, Takahashi, W: Strong convergence of a proximal-type algorithm in a Banach space. SIAM J. Optim. 13, 938-945 (2002)

12. Kohsaka, F, Takahashi, W: Existence and approximation of fixed points of firmly nonexpansive-type mappings in Banach spaces. SIAM J. Optim. 19, 824-835 (2008)

13. Aoyama, K, Kohsaka, F, Takahashi, W: Three generalizations of firmly nonexpansive mappings: their relations and continuity properties. J. Nonlinear Convex Anal. 10, 131-147 (2009)

14. Kohsaka, F, Takahashi, W: Fixed point theorems for a class of nonlinear mappings related to maximal monotone operators in Banach spaces. Arch. Math. 91, 166-177 (2008)

15. Rockafellar, RT: On the virtual convexity of the domain and range of a nonlinear maximal monotone operator. Math Ann. 185, 81-90 (1970)

16. Barbu, V: Nonlinear Semigroups and Differential Equations in Banach Spaces. Editura Academiei Republicii Socialiste România, Bucharest (1976)

17. Takahashi, W: Convex Analysis and Approximation of Fixed Points. Yokohama Publishers, Yokohama (2000) (in Japanese)

18. Rockafellar, RT: On the maximality of sums of nonlinear monotone operators. Trans. Am. Math. Soc. 149, 75-88 (1970)

19. Zălinescu, C: Convex Analysis in General Vector Spaces. World Scientific, River Edge (2002)

20. Rockafellar, RT: On the maximal monotonicity of subdifferential mappings. Pac. J. Math. 33, 209-216 (1970)

21. Brøndsted, A, Rockafellar, RT: On the subdifferentiability of convex functions. Proc. Am. Math. Soc. 16, 605-611 (1965)

10.1186/1029-242X-2014-181

Cite this article as: Kohsaka: An implicitly defined iterative sequence for monotone operators in Banach spaces. Journal of Inequalities and Applications 2014, 2014:181

\section{Submit your manuscript to a SpringerOpen ${ }^{\circ}$ journal and benefit from:}

- Convenient online submission

- Rigorous peer review

- Immediate publication on acceptance

- Open access: articles freely available online

- High visibility within the field

- Retaining the copyright to your article 